\title{
Preferential Spectral Image Quality Model
}

\author{
D. Kalenova, P. Toivanen, and V. Bochko \\ Spectral Image Processing and Analysis Group, Laboratory of Information Processing, \\ Department of Information Technology, Lappeenranta University of Technology, \\ P.O.Box 20, 53851 Lappeenranta, Finland \\ \{Diana.Kalenova, Pekka.Toivanen, Vladimir.Botchko\}@lut.fi
}

\begin{abstract}
In this paper a novel method of spectral image quality characterization and prediction, preferential spectral image quality model is introduced. This study is based on the statistical image model that sets a relationship between the parameters of the spectral and color images, and the overall appearance of the image. It has been found that standard deviation of the spectra affects the colorfulness of the image, while kurtosis influences the highlight reproduction or, so called vividness. The model presented in this study is an extension of a previously published spectral color appearance model. The original model has been extended to account for the naturalness constraint, i.e. the degree of correspondence between the image reproduced and the observer's perception of the reality. The study shows that the presented preferential spectral image quality model is efficient in the task of quality of spectral image evaluation and prediction.
\end{abstract}

\section{Introduction}

The nature and the scope of imaging have been undergoing dramatic changes in the recent years. The latest trend is the appearance of multiprimary displays and printers that reproduce images with a closer spectral match to the original scene captured $[1,2]$. Appearance of such devices gives rise to a problem already existing for conventional tools - assessment of perceptual image quality given only physical parameters of the image. The demand for a quantitative analysis of image quality has dramatically increased. Preferential spectral image quality model, presented in this work is intended to create a paradigm that would allow description of the quality of spectral images in terms of objectively measurable parameters of spectral images in connection with the subjective quality metrics. The preferential spectral image quality model, presented in this paper, can be used for spectral image quality evaluation and prediction in tasks of e.g. imaging device production and calibration, printing industry and in some other industrial applications.

The model described in this paper is based on the results of a previously published spectral color appearance model. The model, introduced in [3], has been used for color image quality estimation, with a color image, reproduced through the spectral image. Spectral color appearance model has demonstrated that there is a close connection between the quality judgments of the observers and the parameters of the model, i.e. vividness and colorfulness, which in turn have been proven to depend upon statistical characteristics of spectral images, in particular, standard deviation and 
kurtosis. This corresponds with the results obtained by other researchers [4]. Fedorovskaya and de Ridder in [4] suggest that scaling of the perceived strength of colorfulness impression is linearly related to the average and standard deviation of the chroma variations in CIELUV color space.

In every set of images produced through variation of both parameters of the spectral color appearance model, one image has been found to be of maximal quality, meaning that it had the highest quality judgment given by the observers. Moreover, there has been found a significant difference between the quality judgments of the scenes, meaning that part of the images exhibited a clear maximum at points close to the original scenes, whilst the others have had a significant shift in the highest quality judgment position. This model has already proven to be effective in the task of image quality evaluation, however it lacks universality, in a sense, that units of quality used are artificial and have weak mathematical basis, which, in turn, does not allow comparison with analogous reference systems. Another serious drawback is that modeling of a combined effect of the parameters of the model on the overall quality impression has been reproduced via Fuzzy Inference System, and has resulted in serious error rates in some cases [3].

In general, the spectral color appearance model and the preferential spectral image quality model, introduced in this paper, can be attributed to a class of preferential quality models. A number of publications exist on the topic of preference in color and tone reproduction in the framework of image quality investigation [5, 6, 7]. Among the whole range of preferential characteristics, contrast, saturation and memory color reproduction are the most common ones. These features change is evident in the image and is highly dependent upon the observer and the content of the scene. Normally, such image attributes have an optimal value where the overall impression is most pleasing [7].

Spectral color appearance model has been created upon the assumption that colorfulness and vividness can efficiently describe image quality, with colorfulness including both contrast and saturation. Previous research is extended in this work to account for memory color reproduction or as it will further be called naturalness. The naturalness constraint imposed upon the image quality stems from an intuitive assumption that high quality images should at least be perceived as "natural". At base, this assumption rests on the psychological model of the quality judgment constitution. Accordingly, the impression of an image is formed as a result of comparison of the output of the process of visual perception and the internal representation of an object, which, in turn, is based on the previous experience (i.e. memory representation or prior knowledge of reality). That is, a high quality image complies with the ideas and expectations of the scene captured in the image at hand. Several works exploring the influence of naturalness on color image quality exist at the moment $[4,8]$, particularly, in the field of color photography. A direct dependence between the naturalness constraint and the quality judgments has been experimentally found in these, with memory colors being relatively consistent among different observers. However, for the case of colorfulness variation a discrepancy between the naturalness judgments and the perceived quality has been found, i.e. observers perceived more colorful images as being of higher quality, at the same judging these images as unnatural. This phenomenon can be explained from the information-processing point of view, a high degree of naturalness is a necessary, but not a sufficient condition for 
quality perception, a usefulness condition has to be satisfied as well, which, in turn, leads to a discriminability principle. In other words, a highly saturated image is perceived to be of high quality, despite being unnatural, due to an increased possibility of discerning certain features in an image $[4,8]$. In this study we are trying to establish a connection between quality judgments of spectral images, spectral image attributes and the naturalness constraint with regard to the principles mentioned.

\section{Statistical Model}

A generalized statistical model, characterizing the behavior of statistical characteristics of natural spectral images $\mathbf{f}(\mathbf{x})$, presented as n-dimensional vector random field, is described by the following equation [9]:

$$
\mathbf{f}(\mathbf{x})=\mu+\operatorname{Dg}(\mathbf{x})
$$

where $\mathbf{x}$ is a vector with each element being spatial dimension; $\boldsymbol{\mu}$ is a mean vector, $\mathbf{g}(\mathbf{x})$ is a normalized vector image with zero mean and unit standard deviation for each component, $\mathbf{D}=\operatorname{diag}\left(\sigma_{1}, \sigma_{2}, \ldots, \sigma_{\mathrm{n}}\right)$, where $\sigma_{\mathrm{i}}$ is standard deviation in the component [3]. The following parameters: $\alpha, \beta$ and $\mathrm{k}_{\max }$ are used for modifying the colorfulness and vividness change in the experiment.

Vector $\sigma$ is presented in the following form:

$$
\sigma=\alpha \beta \sigma_{\mathrm{v}}+(1-\alpha) \sigma_{\mathrm{c}}
$$

where $\alpha=\left(\sigma_{\max }-\sigma_{\min }\right) / \sigma_{\max }$ is the relationship between constant and variable parts of standard deviation, affecting the saturation of colors in an image, and $\beta$ is a contrast variation coefficient, $\sigma_{\mathrm{v}}$ is a variable component vector of $\sigma, \sigma_{\mathrm{c}}$ is a constant component vector of $\boldsymbol{\sigma}[3]$.

$\mathbf{g}(\mathbf{x})$ is defined through gamma-Charlier histogram transform of $\mathbf{f}_{\mathrm{s}}(\mathbf{x})$ and a kurtosis vector $\mathbf{k}$ as follows [3]:

$$
\mathbf{g}(\mathbf{x})=\mathbf{H}\left(\mathbf{f}_{\mathrm{s}}(\mathbf{x}), \mathbf{k}\right)
$$

where $\mathbf{f}_{\mathbf{s}}(\mathbf{x})$ is a normalized image of $\mathbf{f}(\mathbf{x})$, with zero mean and unit standard deviation for each component. To affect the image appearance through histogram transform, all kurtosis elements are proportionally modified according to the given maximum of the kurtosis value $\mathrm{k}_{\max }$ [3].

The task of quality manipulation is a complicated task that requires significant computational resources. Spectral images contain large amounts of information, which have to be manipulated in order to influence the overall impression of the display. Usually, some implicit assumptions are made in order to limit the amount of computations. The assumption underlying this study is that only global variations are taken into account, which, in turn, originates from the fact that all parts of the image have been captured under the same illuminant or belong to the same object. Thus the same modifications are applied to all pixels of the image irrespective of the content [4]. Based upon this principle the generalized statistical model is applied to spectral images in this study. 


\section{Experiment}

Experiments were performed on spectral images of natural scenes from [10]. Five images - inlab1, inlab2, inlab5, jan13am and rleaves were selected (see Fig.1). Images have the following dimensions: 256x256 pixels, and 31 spectral components per each pixel. For the purpose of the experiments the area of $128 \times 128$ pixels were selected. Images were captured by a CCD (charge coupled device) camera in a 400$700 \mathrm{~nm}$ wavelength range at $10 \mathrm{~nm}$ intervals. The images selected were taken indoor (in a controlled environment, i.e. dark-lab or glass-house).

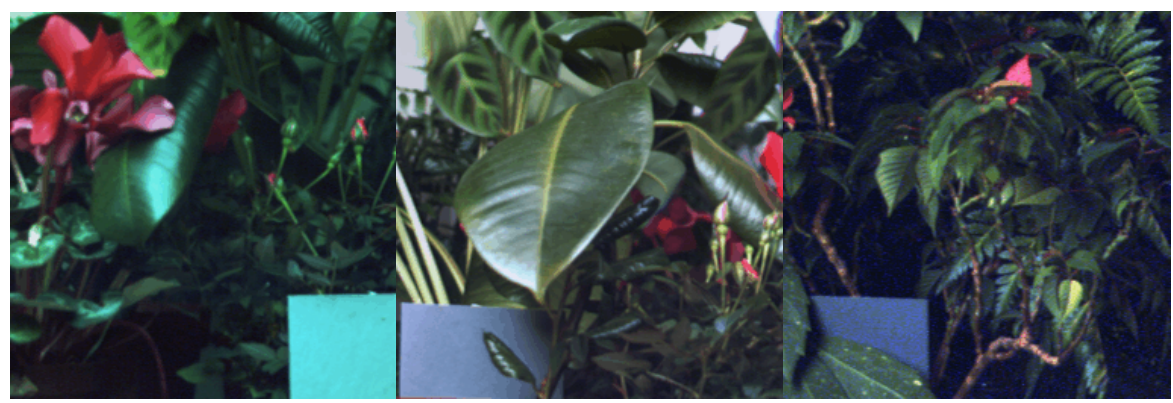

(a)

(b)

(c)

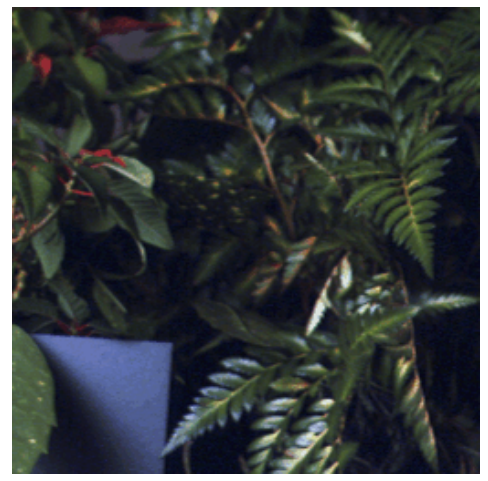

(d)

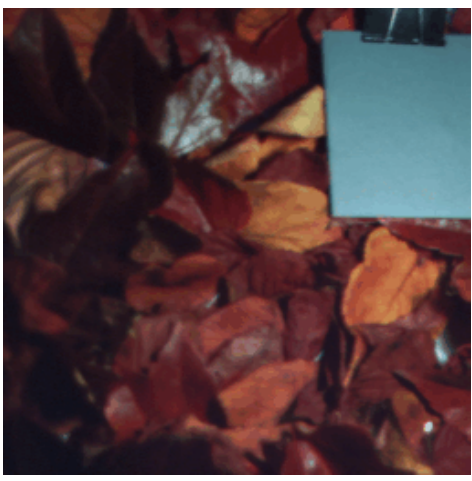

(e)

Fig. 1. Color reproduction of original spectral images used in the experiments (a) inlab1, (b) inlab2, (c) inlab5, (d) jan13am, (e) rleaves

Experimental settings, which include the number and the criteria for selection of observers, test stimuli, instructions and viewing conditions requirements, were chosen to comply with [11]. According to this standard relative quality values should be obtained from at least ten observers and three scenes, and all of the observers have to be tested for normal vision and visual acuity. Thus, we chose twenty observers to participate in the experiments. They had normal or corrected-to-normal vision without color deficiencies. To prevent the loss of quality of judgments due to fatigue the duration of the experimental sessions was limited to one hour, in case of more time needed the experiments continued after a break. Viewing conditions followed the 
requirements given in [12]. Therefore, the general room lighting was shaded and was set so that it neither directly nor indirectly influenced the viewing surface. When viewing slides, the frames were darkened to a $10 \%$ brightness level for a width of $75 \mathrm{~mm}$.

Although original images were presented, it should be emphasized that they were not explicitly identified to observers as such. First, a set of test images was produced using the colorfulness parameter. The term includes both contrast and color saturation. Thus, colorfulness was varied through standard deviation, using Eq.2. By changing $\alpha$ and $\beta$ coefficients it was possible to receive new values for constant and variable parts of standard deviation. This procedure was applied to the images with values of $(\alpha, \beta)$ equal to $(0.55,1),(0.75,1),(1,1.3),(1,1.6)$ consequently. The second set of tests was produced through variation of the vividness parameter, closely related to highlight reproduction in an image. As the highlight was modified through kurtosis change, the test images were produced with the help of Eq.3 (with $\mathrm{k}_{\max }$ equal to 5, 10, $30,60)$. The effect of the change of both of parameters on the overall appearance had been shown in [3]. Both test sets were presented to the subjects, who had to rate the naturalness of the images on a ten-point numerical category scale ranging from one (unnatural) to ten (real life). The following instructions for the experiments were given to the observers [4]:

"You will be presented a series of images. Your task is to asses the naturalness of images, using and integer number from one to ten, with one corresponding to the lowest degree of naturalness and ten to the highest. Naturalness is defined in this case as the degree of correspondence between the image reproduced on the screen and reality, i.e. the original scene as you picture it."

The results of the tests are given in Table 1, where each cell corresponds to an averaged naturalness evaluation score, with outliers being excluded from consideration. The columns denoted as 1 present results of the tests produced through the colorfulness change, and 2 with the vividness change respectively. In Table 1 Image 1 and Image 2 have parameters $\left(\alpha, \beta, \mathrm{k}_{\max }\right):(0.55,1,5),(0.75,1,10)$, Image 3 is the original, Image 4 and Image 5 have respectively parameters $\left(\alpha, \beta, \mathrm{k}_{\max }\right)$ equal to $(1,1.3,30),(1,1.6,60)$. Note that either $(\alpha, \beta)$ (for colorfulness change) or $\mathrm{k}_{\max }$ (for vividness change) were varied, while the rest of the parameters were kept constant.

Table 1. Mean values of naturalness evaluation scores

\begin{tabular}{lllllllllll}
\hline Quality & \multicolumn{3}{c}{ Image 1 } & \multicolumn{3}{c}{ Image 2 } & \multicolumn{3}{c}{ Image 3 } & \multicolumn{2}{c}{ Image 4 } & \multicolumn{2}{c}{ Image 5 } \\
\hline & 1 & 2 & 1 & 2 & 1 & 2 & 1 & 2 & 1 & 2 \\
\cline { 2 - 12 } Inlab1 & 2.37 & 5.41 & 5.83 & 6.34 & 8.12 & 8.34 & 9.67 & 9.83 & 8.15 & 9.12 \\
Inlab2 & 2.25 & 6.03 & 6.93 & 7.98 & 8.93 & 9.67 & 9.87 & 5.56 & 8.41 & 3.98 \\
Inlab5 & 3.84 & 7.34 & 6.55 & 7.53 & 8.34 & 9.12 & 9.85 & 9.53 & 8.56 & 9.34 \\
Jan13AM & 3.10 & 6.56 & 7.12 & 8.96 & 8.67 & 9.87 & 9.73 & 6.76 & 8.17 & 4.87 \\
Rleaves & 3.32 & 6.17 & 6.86 & 9.10 & 8.16 & 8.17 & 9.54 & 7.65 & 9.50 & 5.35 \\
\hline
\end{tabular}

Looking at Table 1 we can state that the peaks of the naturalness judgments do not lie within the original image area, which in turn brings us to a conclusion that users generally prefer slightly modified images. Fig. 2 illustrates the connection between 
the naturalness constraint and statistical parameters of the spectral images varied at the experiments.

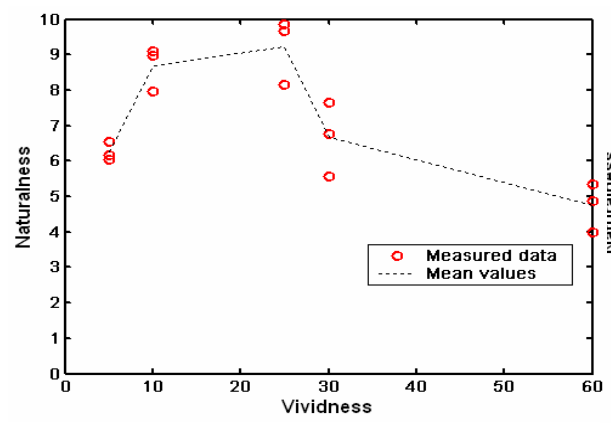

(a)

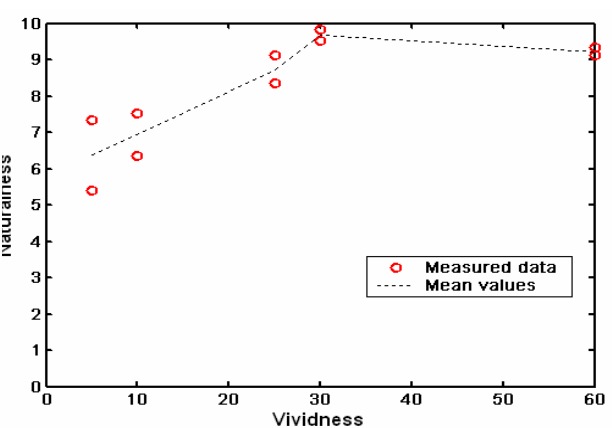

(b)

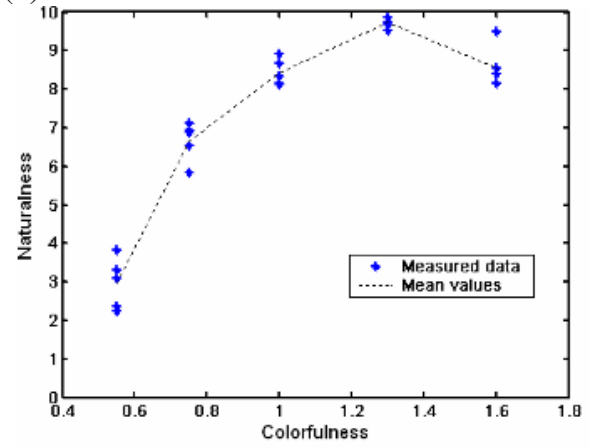

(c)

Fig. 2. Averaged naturalness estimations vs. statistical parameters of spectral images. Vividness (a) of inlab2, jan13am, rleaves; vividness (b) of inlab1 and inlab5; colorfulness (c) of all of the images

Fig. 2 demonstrates that there is positive correlation between the attributes of spectral images and the naturalness constraint. Fig. 2 (a) and (b) specifically present the relation between the vividness spectral image attribute and the naturalness constraint. It is clearly visible that the naturalness maximum lies at points close to the original image. We have separated the vividness parameter versus naturalness plots onto two parts due to a different form of dependency between the two. Fig. 2(a) contains a plot of the inlab2, jan13am, rleaves image judgments, and Fig. 2(b) inlabl and inlab5. In the first case images exhibit a sharper drop in the naturalness judgments than in the second one, in fact, in the second case the decrease in naturalness is such that the naturalness remains approximately close to the maximal value. Such discrepancy in the image judgments has also been obtained when evaluating the quality of the images [3]. Both of the phenomena can be attributed to a fact that images inlabl and inlab5 contain objects that attract the most of the observers' attention, compared to the objects situated at the background. Moreover, these objects lie in the red area of the spectrum, which assumes that observers are not 
susceptible to minor variations in these areas due to the properties of the human visual system. Thus, the drop in quality and in naturalness is less definitive.

Fig. 2(c) demonstrates the connection between the colorfulness parameter and the naturalness constraint. It can be stated that observers perceive slightly more colorful than original images as being the most natural ones. This effect is consistent with the results obtained in the experiments with color images, stating that there is a tendency for memory colors to be slightly more saturated compared with actual object colors [13]. Moreover, considering the fact that observers have previously rated the images with higher colorfulness values as being of higher quality [3] we can state that memory color reproduction influences the preferred color reproduction of the objects [14].

Another important characteristic of image naturalness is correlation with the quality perception. For this purpose the quality judgment values have been taken from the previous study [3] and plotted against the naturalness obtained in this study. Fig. 3 demonstrates a plot of the quality judgments versus the naturalness constraints for both vividness (red circles) and colorfulness (blue asterisk) test sets. Such a comparison is possible due to the fact that experimental settings (number of observers, number and contents of scenes, viewing conditions, etc.) are similar in both of the experiments, moreover the algorithm of modification and values of the statistical parameters of spectral images are the same.

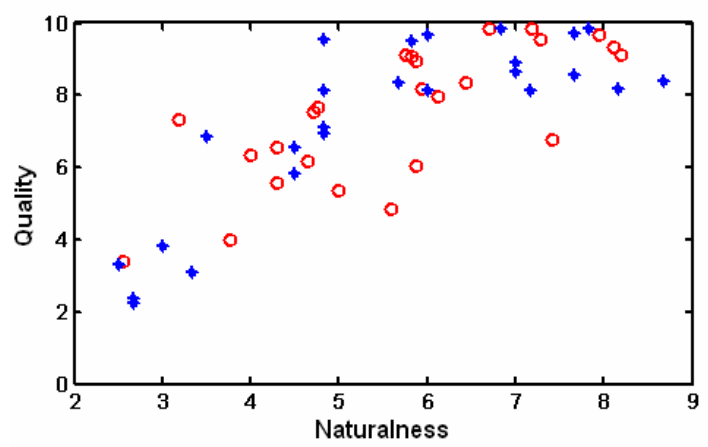

Fig. 3. Averaged naturalness estimations vs. perceptual quality estimations of images with the vividness (red circles) and colorfulness (blue asterisk) change

Fig. 3 illustrates the interdependence between the quality judgments and the naturalness constraint. It is clear that there is a strong correlation between these notions. In fact, the correlation coefficient between the quality judgments and naturalness estimations computed over all of the scenes equals to 0.8196 for colorfulness, 0.7018 in the case of vividness test sets, and the overall correlation is equal to 0.7752 .

With the increase of naturalness the quality also increases, which proves the preliminary assumption made, stating that in order for the image to be of good quality it should at least be perceived natural. The spread in the plot can be attributed to a lack of test images and a rough scale of spectral image attributes accepted in this 
study. However, even such a small test set was enough to prove a connection between the attributes of spectral images, naturalness and overall image quality impression.

Even though we can see from the plot in Fig. 3 that there is a connection between the naturalness constraint and the quality judgments of the users, it is relatively difficult to predict what would be the effect of the naturalness change on image quality, and how fast does the quality decrease with the decrease in naturalness, which in turn can be varied through variation of any of the attributes of spectral images. Accordingly naturalness could serve as a universal image attribute that would allow modeling both image quality and the joint effect of attributes of spectral images on the overall perception of the image reproduction. In order to model the effect of naturalness on quality a preference distribution function of naturalness expressed in terms of JNDs has been constructed. According to [11] JND is a stimulus difference that yields a $75 \%: 25 \%$ proportion in a forced-choiced paired comparison. In other words, JND is the smallest possible difference between two stimuli, noticeable by observers. The standard distinguishes two types of JND units, attribute and quality JNDs. In our study we have used the quality JNDs, which is a measure of the significance of perceived differences on overall image quality. Essentially all of the observers could detect the difference and identify which of the samples have had higher naturalness.

The preference data was obtained with the use of the rank order technique. The observers were presented small sets of stimuli (5 images at a time) and had to rank the images according to the quality of these. The result of such experiments is noisier than in a paired-comparison technique, however it significantly reduces sample handling. To construct the quality preference function value of each of the attributes of the highest rated image was identified for each scene and observer, and the fraction of times each position had been preferred was computed. To convert each fraction to a probability density, it was divided by the width of an attribute interval including the sample position. The resulting preference distribution function is presented in Fig. 4.

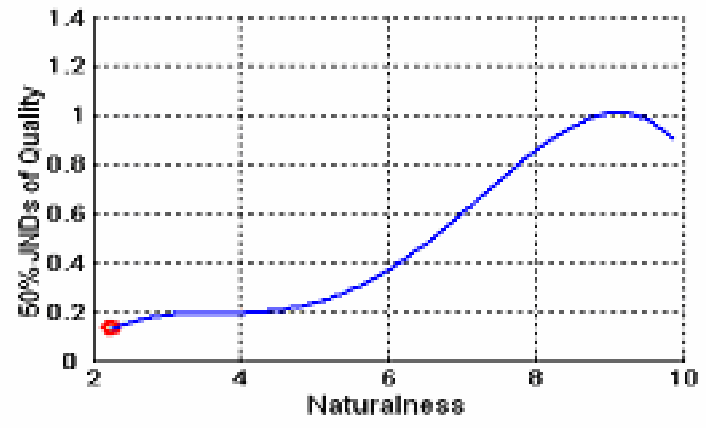

Fig. 4. Averaged preference distribution function of naturalness

A conclusion conforming to the preliminary assumption imposed upon the naturalness constraint can be drawn based on Fig. 4: with the increase of naturalness the quality of the images increases. However, at a certain point naturalness starts to decrease. Thus, high degree of naturalness is a necessary, but not a sufficient 
condition for quality perception. A usefulness condition has to be satisfied as well, which in turn leads to a discriminability principle. Meaning that, even if the image gives an impression of being unnatural, it might be perceived as being of high quality, due to the fact that the information in the image is easily discriminable. Therefore, naturalness has a strong connection with the statistical characteristics of spectral images and image quality perception on the whole, being a necessary, but not a determinative factor.

\section{Conclusions}

In this paper, a preferential spectral image quality model has been presented. The model sets a relationship between the statistical characteristics of spectral images, overall quality, and perceived naturalness. The model described in this paper is an extension of a previously published spectral color appearance model. The original study has been extended to account for the naturalness constraint, i.e. the degree of correspondence between the image reproduced and the observers' perception of the reality.

Several conclusions can be drawn upon this study. One of the important inferences drawn from this work is that a strong connection between the statistical parameters of spectral images and the naturalness perception does exist. Particularly, not only there is a strong correlation between the colorfulness parameter, but it can also be said that there is a tendency for memory colors to be slightly more saturated compared with actual object colors, meaning that observers perceive slightly more colorful than original images to be more natural [13]. Moreover, considering the fact that observers generally discern more colorful images as being of higher quality, it can be stated that memory color reproduction influences the preferred color reproduction of the objects [4].

The connection between the vividness parameters and naturalness is twofold. Part of the images used in the experiment exhibit a sharper drop in the naturalness judgments than the rest, in fact, in the second case the decrease in naturalness is such that the naturalness remains approximately close to the maximal value. A similar phenomenon has been found in the spectral color appearance model [3] concerning image quality judgments. Both of the phenomena can be attributed to a fact that images inlabl and inlab5, that exhibit an insignificant drop in both of the characteristics compared with the rest of the images, contain objects that attract the most of the observers' attention, in comparison with the objects situated at the background. Moreover, these objects lie in the red area of the spectrum, which assumes that observers are not susceptible to minor variations in these areas due to the properties of the human visual system. Thus, the drop in quality and in naturalness is less definitive.

The last conclusion is the connection between the naturalness constraint and the overall perceived image quality. Although with the increase in naturalness the quality of the images increases, naturalness of the image is a necessary, but not a sufficient condition for the high quality judgments. A usefulness condition has to be satisfied as well. Thus the peak of the quality judgments does not lie at the highest naturalness value, meaning that observers knowing that the image is unnatural would still 
perceive the image as being of high quality. For the purpose of modeling the naturalness influence upon image quality a preference distribution function, describing the impact of the naturalness on the quality judgments in terms of JNDs has been constructed. The function can be used for spectral image quality prediction in terms of image naturalness.

In general, both the preferential spectral image quality and the spectral color appearance models can be attributed to a class of preferential image quality models and can serve as an efficient tool of image quality characterization and prediction.

\section{References}

1. Hardeberg, J. and Gerhardt, J.: Characterization of an Eight Colorant Inkjet System for Spectral Color Reproduction, in Procs. Second European Conf/ on Colour Graphics, Imaging and Vision, Aachen, Germany (2004) 263-267.

2. Rosen, M., Hattenberger, E. and Ohta, N.: Spectral Redundancy in a 6-ink Inkjet Printer, in Procs. of The Dig. Phot. Conference, Rochester, NY, USA (2003) 236-243.

3. Kalenova, D., Botchko, V., Jaaskelainen, T. and Parkkinen, J.: Spectral Color Appearance Modeling, in Proc. Dig. Phot. Conference, Rochester, NY, USA (2003) 381-385.

4. Fedorovskaya, E.A., de Ridder, H. and Blommaert, F.J.J.: Chroma Variations and Perceived Quality of Colour Images of Natural Scenes, J. Color res. and appl. 22 (1997) 96-110.

5. Buhr, J.D. and Franchino, H. D.: Color Image Reproduction of Scenes with Preferential Tone Mapping, U.S. Patent \#5 447 (1995) 811.

6. de Ridder, H.: Saturation and Lightness Variation in Color Images of Natural Scenes, J. Imaging Sci. and Techn. 6(40) (1996) 487-493.

7. Janssen, R.: Computational Image Quality, (2001) 20-35.

8. de Ridder, H.: Naturalness and Image Quality: Saturation and Lightness Variation in Color Images of Natural Scenes, J. Imaging Sci. and Techn. 40 (1996) 487-498.

9. Botchko, V., Kälviäinen, H. and Parkkinen, J.: Highlight Reproduction Using Multispectral Texture Statistics, Proc. Third Int. Conf. on Multispectral Color Science, Joensuu, Finland (2001) 61-65.

10. Parraga, A., Brelstaff, G. and Troscianko, T.: Color and Luminance Information in Natural Scenes, J. of Opt. Soc. of America A 15 (1998) 3-5.

11. ISO/DIS 20462-1, Psychophysical Experimental Method to Estimate Image Quality - Part 1: Overview of Psychophysical Elements, International Organization for Standardization (2003).

12. ISO 3664, Graphic Technology and Photography: Viewing conditions, International Organization for Standardization (2000).

13. Newhall, S. M., Burnham, R. W. and Clark, J. R.: Comparison of Successive with Simultaneous Color Matching, J. of Opt. Soc. of America 47 (1957) 43-56.

14. Siple, P., and Springer, R. M.: Memory and Preference for the Color of Objects, Perception and Psychophysics 33 (1983) 363-370. 